\title{
Accuracy of Bit Error Probability for W-CDMA System Using Code Tree
}

\author{
Anwar Hassan Ibrahim \\ Department of Electrical Engineering \\ Qassim University, College of Engineering \\ Buraydah, Saudi Arabia \\ dr.anwar@qec.edu.sa
}

\begin{abstract}
W-CDMA is radio access utilized for 3G cell frameworks. A code tree allocation scheme is one of the most explored channelization techniques, used to improve system performance and capacity through adjustable data rates. This work investigates the accuracy of bit error probability for WCDMA system using code tree orthogonal variable spreading factor (OVSF) codes, compared to pseudo-noise (PN) codes under various noise conditions, such as additive white Gaussian Noise (AWGN) and Random Noise (RN). Results are carried out theoretically and by computer simulation. The simulation includes the scenario of simple model representation for $\mathrm{W}$ CDMA system. It was concluded that, the system has better performance using OVSF compared with PN code under different noisy channels.
\end{abstract}

Keywords-W-CDMA; OVSF code; PN code; $A W G N ; R N$; bit error probability (BEP)

\section{INTRODUCTION}

Data spreading in W-CDMA systems is done by the application of a signal independent code. The code choice affects the framework execution. The more extended the code, the higher the preparations picked up, which empowers the system to permit more clients in the framework. Then again, a bigger preparation, infers the use of more transfer speed in $\mathrm{W}$ CDMA [1]. A good W-CDMA planning model for communications development reduces the complexity of solutions in order to integrate them into the same channel by using code division multiple access (CDMA), data, and web services. 3G wireless standards use W-CDMA to meet high data rates and variable rate requirements. The proposed scenario initially attempts to assign request codes to the system, and then tries to allocate them to user access. In order to achieve high bit error rate accuracy [2, 3], different data must be used by different user connections with variable operation rates and OVSF. In terms of implementation, it is better to have different spreading factors from the same branch of the tree to avoid chip level buffering [4]. The more the channel is used, the more noise is produced [5]. Furthermore, the scenario studies the efficiency performance of OVSF code tree in WCDMA system under two different data under noise comparing to PN code. W-CDMA is a flexible system, supporting variable data rates and services. The flexibility of using OVSF as channelization code increases the ability of supporting variable data rates for each transceiver and makes simpler hardware usage [6].

\section{OVSF CODE TREE}

\section{A. Orthogonality}

The tree structure code method is usually assigned to certain users with different data rates orthogonally. The design of OVSF codes has different lengths on different levels and different spreading factors, related to the information rate multiplied by the entire code word [7]. Two codes are supposed to be orthogonal when their inner product is zero. For example, the characterized code of $(1,1,1,1)$ and $(1,1,-1,-1)$ are orthogonal. Their product is zero as shown below:

$$
(1 * 1)+(1 * 1)+(1 *-1)+(1 *-1)=0
$$

\section{B. Code Tree Definition}

OVSF codes have been introduced for $3 \mathrm{G}$ communication systems. Spectrum spreading is attained by plotting each bit (1 or -1) into an allocated code categorization. Figure 1 shows the tree structure [8]. OVSF codes are continuously powering data rates with respect to the lowest simple rate. The potential rates supported are: $\mathrm{Rb}, 2 \mathrm{Rb}, 4 \mathrm{Rb}, 8 \mathrm{Rb}$, etc, with $\mathrm{Rb}$ meaning "rate bit", and the break becomes greater as the rate growths. In particular cases it may over-serve by a greater rate [6].

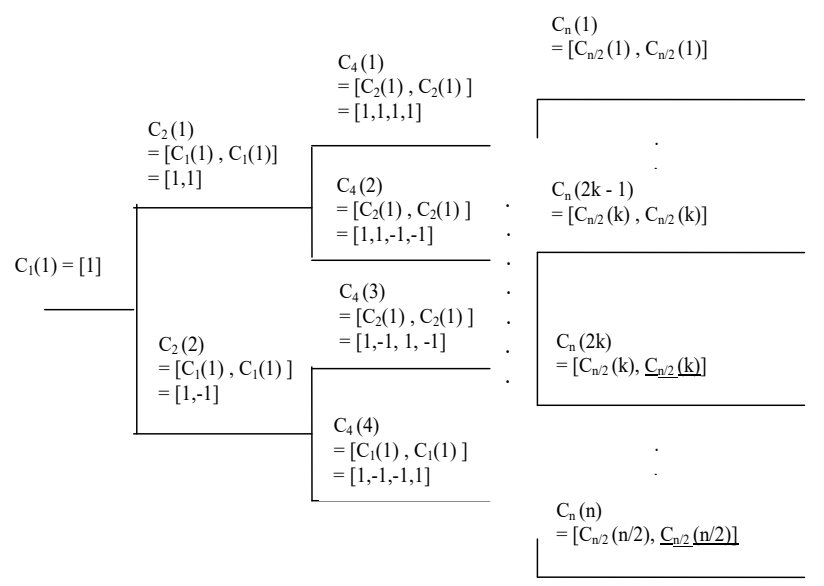

Fig. 1. OVSF code structure 


\section{Code Tree Algorithm}

Different distribution code factors, means different code extents. The general idea is to enable the merging of changed messages with alternative spreading factors and retain their orthogonality. Code dimensions are needed to be orthogonal. The analysis below shows the workability of the algorithm. The process of the first section in the tree is 1. For each level, there are two conceivable sub-levels, represented as top and bottom sub divisions. The top sub division is constructed by repeating the root of the sub-division twice. So in this case the top sub-section of (1) would be $(1,1)$ and the bottom subdivision is assembled by self-inverse of (1), and it would be (1, $-1)$. At each section, all the codes are the rows of a Hadamard matrix with the fundamentals mapped to polar arrangement. The type of code tree depends on the chosen code through the design in Figure 2. If $\mathrm{SF}=8$, the other level can't be used [9].

\section{PN-CODE ALGORITHM}

A pseudo-noise (PN) used for direct sequence spreading code consists of NDS components, named chips. These chips require 2 values: either $-1 / 1$ or $0 / 1$. The bit classifications are used unless specified otherwise. Each data symbol is mutual with a single comprehensive PN-code, the direct sequence is identical to the code-length. PN sequences are periodic structures that have similar behavior with noise [10]. This code is generated by using shift registers, module adders represented by XOR gates and feedback loops. Figure 2 shows the scenario for generating PN code.

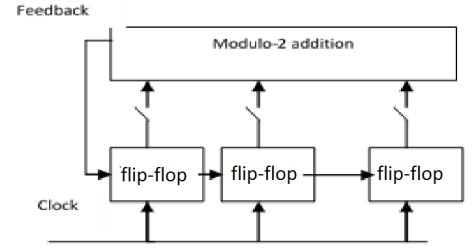

Fig. 2. Shift register of PN code generation

The extreme dimension of a PN sequence is defined by the size of the register and the structure of the feedback system. An $\mathrm{N}$ bit sequence created can consist of up to $2 \mathrm{~N}$ different groupings of zeros and ones. Meanwhile the feedback system achieves linear processes, if all the inputs of the flip-flops are zero, the result of the output from the feedback system will also be zero. Consequently, the entirely zero grouping will always stretch zero output for all following clock cycles, so the system avoids including it in the sequence. Hence, the determined length of several PN sequences is $2 \mathrm{~N}-1$ and classifications of that extent are called maximum-length arrangements or $\mathrm{m}$ sequences. This became preferable. Feedback outlines for $\mathrm{m}-$ sequences are organized and can be found in functions created in Matlab. The signal is usually multiplied by a PN code. A PN code is a classification of chips valued as -1 and 1 (polar sequence) or 0 and 1 (non-polar sequence) and has similar noise properties. To create a PN code in the proper way, at least one shift-register should be actively fast. As long as the length of the code in such a shift-register is $n$ equal, the result in (1) will represent the period NDS of the above mentioned scenario for the length of the code:

\section{$\mathrm{NDS}=2 \mathrm{n}-1$}

This code is used to determine the frequency spectrum that the produced signal will lodge. It regulates and controls the spreading arrangement of the system.

\section{PROPOSED SYSTEM}

The system model is illustrated in Figure 3.

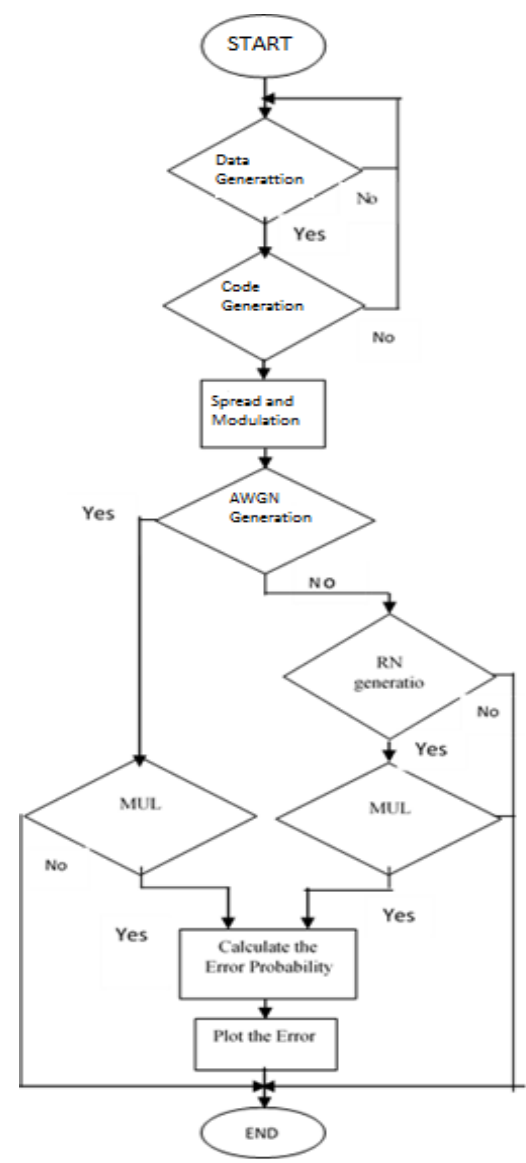

Fig. 3. Illustrattion fo the proposed system model

This section details the developed simulation methodology to evaluate the performance of OVSF code tree. The simulation results demonstrate the code performance for several configurations in different channel environments, which are given. The system consists of two channels where users fed their data into a diversity receiver [11]. In the receiver, the transmitted data are recovered and checked for errors [12]. The simulation study investigates a scenario with undesired channel condition, aiming to examine the refusal of the OVSF code tree to accept this condition and to calculate the probability of error.

\section{BIT ERROR PROBABILITY}

An important application of spread spectrum structures is multiple access infrastructures, in which several users have to access the channel [13]. The probability of error performance in the receiver part is presented. The antenna component separation and the functioning environment parameters (such as random noise (RN) and additive white Gaussian Noise 
(AWGN) generation), in overall space-path multiplicity expanse can be directly assessed. Every one of spreading waveforms is assigned to one equivalent bit vectors. Consequently, each independent message bit to be transferred on the $\mathrm{n}$-th signaling interval is allocated to a defined transmit antenna. The number of $\mathrm{n}$-th user's data transmitted by transmit antenna $\mathrm{k}$ on the $\mathrm{n}$-th signaling is multiplied by spreading waveform $\boldsymbol{w}_{m i}^{(\boldsymbol{n})}$. The composite channel improvement among transmit antenna $\boldsymbol{k}$ and receive antenna $\boldsymbol{j}$ on the n-th signaling interval $\propto_{i j}^{(n)}$. Different users are allocated single sets of spreading waveforms [14]

$$
\mathrm{w}_{\mathrm{mi}}^{(\mathrm{n})}=\mathrm{C}_{\mathrm{mx}}(\mathrm{t}-\mathrm{nT})
$$

$$
\text { for } i=1,2, \ldots . n_{\mathrm{t}} \text { and }(\mathrm{Cmx}=\varnothing \text { when } \mathrm{m} \neq 1)
$$

The designation for these decision variables and how they are joined together is the subject of the spreading technique used by the transmitter. The $k$ th matched filter output on receive antenna $j$ and signaling interval $n$ is $u_{l k}^{(n)}$ and the $x$ th channel gain matrix, then the decision variables of are given by equation 3 .

$$
\mathrm{u}_{\mathrm{Jk}}^{(\mathrm{n})}=\left\{\begin{array}{lr}
\sum_{\mathrm{i}=1}^{\mathrm{Nt}}\left(\sqrt{E_{\mathrm{b}}} \mathrm{b}_{\mathrm{i}}^{(\mathrm{n})} \propto_{\mathrm{ij}}^{(\mathrm{n})}\right)+\mathrm{n}_{\mathrm{Jk}}^{(\mathrm{n})}, \mathrm{K}=\mathrm{x} \\
\mathrm{n}_{\mathrm{Jk}}^{(\mathrm{n})} & \mathrm{K} \neq \mathrm{x}
\end{array}\right.
$$

In addition, we make the usual assumption that power control is used to enable all users' transmissions that reached the user of interest with the same power. Under these conditions, it can be shown, that the receiver bit error probability can be approximated [15].

$$
P_{E}=Q(\sqrt{S N R})
$$

where

$$
\begin{aligned}
& P_{E}=Q\left(\sqrt{\frac{A^{2} T b}{N o}}\right)=Q\left(\sqrt{\frac{2 E b}{N o}}\right) \\
& S N R=\left(\frac{K-1}{3 N}+\frac{N o}{2 E b}\right)^{-1}
\end{aligned}
$$

In which $\mathrm{K}$ is the number of users and $\mathrm{N}$ is the number of chips per bit (the processing gain). The main idea of this paper is to identify the system implemented to assess the performance of the OVSF Code tree and PN code under channel environment. We are assuming that the same power control can be used by all users. Under these conditions, it provides the receiver bit error probability calculated approximately in simple system built. Therefore, it characterizes the number of chips contained in one data bit. Complex processing gain (PG) required more spreading factor. Orthogonal variable spreading factor code tree does not have the greatest spreading behavior and the process of spreading depends on user data rate. Nevertheless the PN sequences need to have more spreading factor, since their power spectral density is focused on a small number of the selected discrete frequencies.

\section{RESULtS}

Figures 4-5 show the accuracy of the bit error probability. Figure 4 shows the $P_{E}$ versus $E_{b} /$ No for the constant processing gain and varying number of users. It is found that the error is approached for every case shown. For example, if 8 users are active and $\mathrm{P}_{\mathrm{E}}$ of $10^{-2}$ is desired, it cannot be achieved no matter what $\mathrm{E}_{\mathrm{b}} /$ No is used. This is one of the drawbacks of W-CDMA by using code tree or PN code. The average error in W-CDMA system, by using OVSF code tree, will be approximately less than the PN code. It is also found that, with more users and larger processing gain, the more accurate the approximation. The other advantage of OVSF code tree is that the variable data give the user an opportunity to introduce the system as well as if it were in good condition. Figure 4 explains $P_{E}$ versus $E_{b} / N_{o}$ for WCDMA system using PN code. The number of users is 4 and the processing gain $=4$. Figure 5 shows $\mathrm{P}_{\mathrm{E}}$ versus $\mathrm{E}_{\mathrm{b}} / \mathrm{N}_{\mathrm{o}}$ for WCDMA system using orthogonal variable spreading factor under distributed users (the number of users is changing when the processing gained is constant).

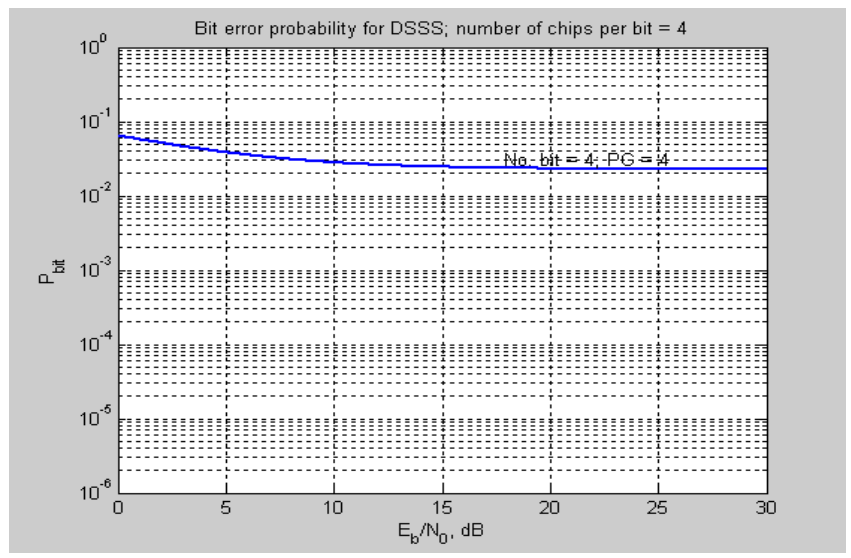

Fig. 4. BEP for the system using PN code

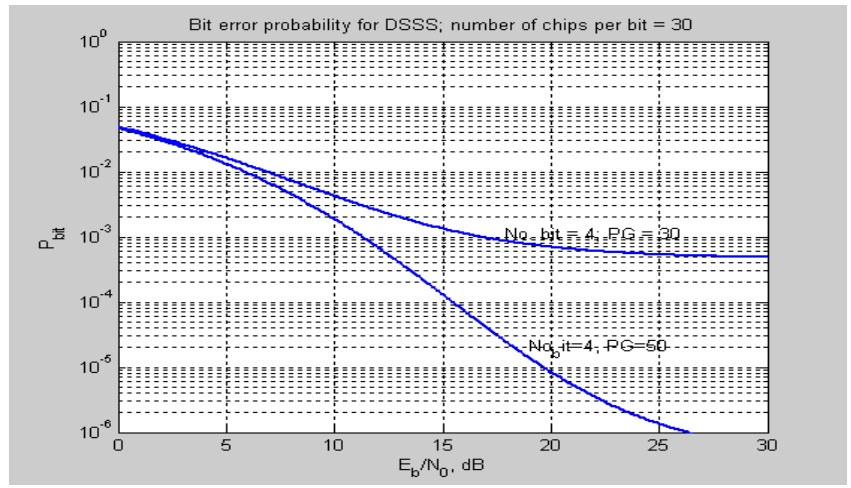

Fig. 5. User distribution for given BEP

Figures 6-9 give detail comparison between the original data transmitted and received for both types of noise (AWGN and $\mathrm{RN}$ ) for OVSF and PN. As a result of adding AWGN to the W-CDMA system with OVSF code, a $25 \%$ error in the transmitted data is detected at the receiver as shown in Figure 6. In Figure 7, RN is added to the W-CDMA system with OVSF code tree and a $18.75 \%$ error occurs. Figure 8 shows a comparison between the original transmitted and received data when adding RN to the W-CDMA system with PN code. In that case a $31.25 \%$ error in the data transmitted was detected at 
the receiver. Adding AWGN to the W-CDMA system with PN code, a $37.5 \%$ error in the data transmitted was detected by the receiver as shown in Figure 9. Table I shows the summarization of data error occurrence in the receiver without filtering and using different codes and different noises.

TABLE I. CLARIFY ERROR OCCURRENCE IN THE RECEIVER

\begin{tabular}{|c|c|c|}
\hline Type of code & Type of noise & Error occur \\
\hline \multirow{2}{*}{ OVSF } & AWGN & $25 \%$ \\
\cline { 2 - 3 } & RN & $18.75 \%$ \\
\hline \multirow{2}{*}{ PN } & AWGN & $37.5 \%$ \\
\cline { 2 - 3 } & RN & $31.25 \%$ \\
\hline
\end{tabular}

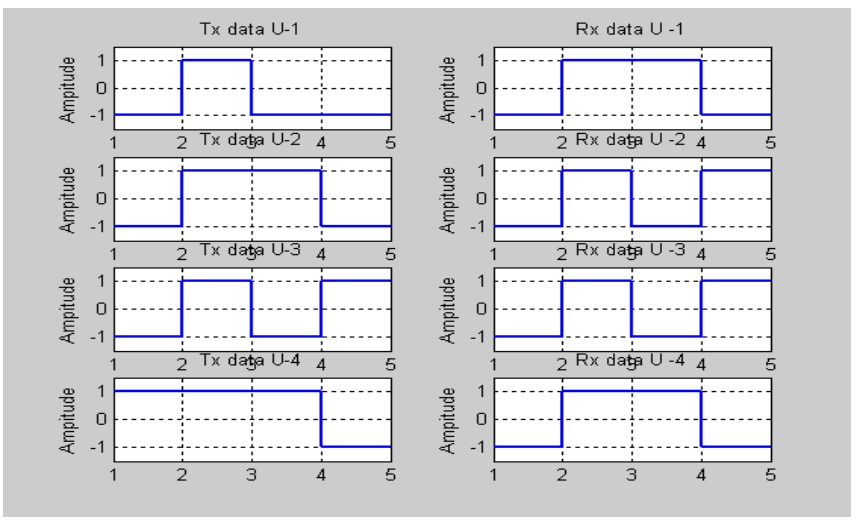

Fig. 6. Data Transmitted vs Data Received for OVSF and AWGN

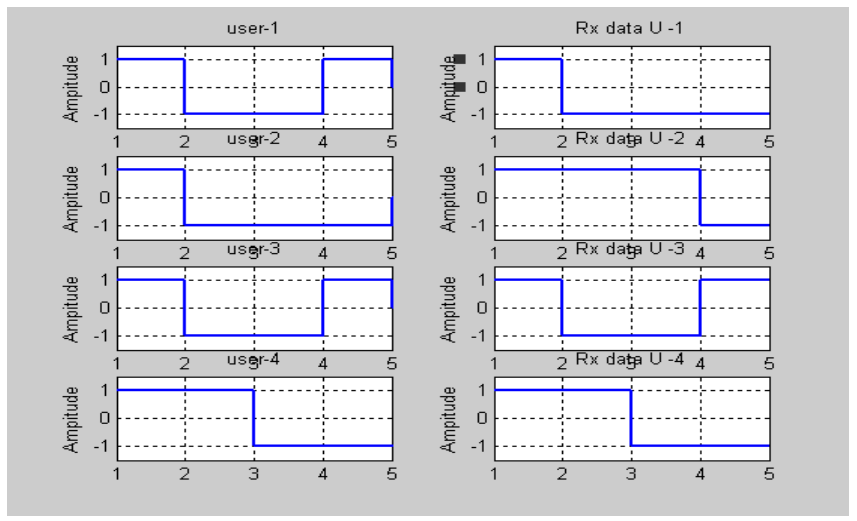

Fig. 7. Data Transmitted vs Data Received for OVSF and RN

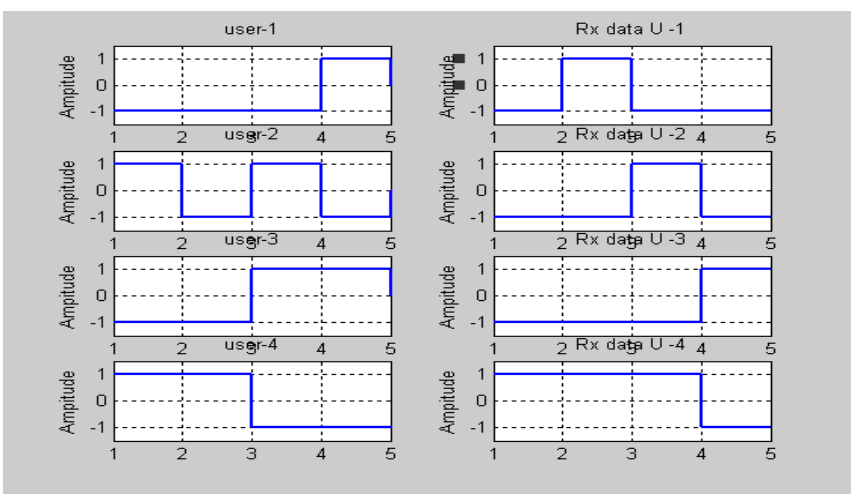

Fig. 8. Data Transmitted vs Data Received for PN and RN
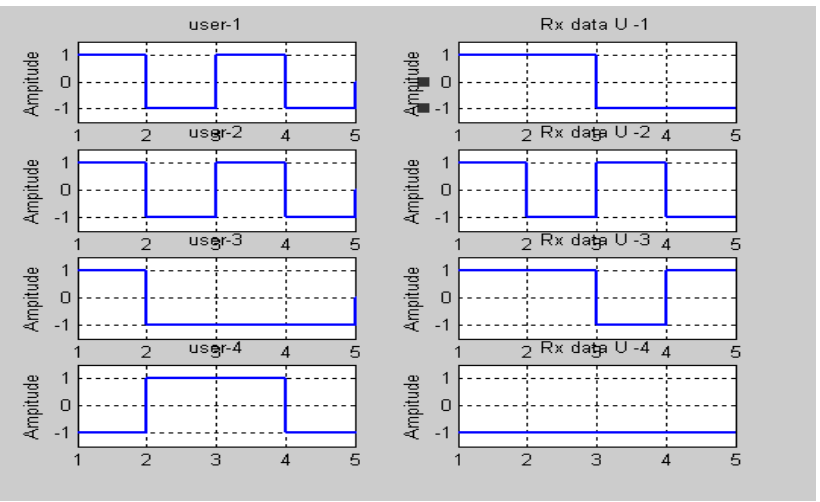

Fig. 9. Data Transmitted vs Data Received for PN and AWGN

\section{CONCLUSION}

Results have shown that in the orthogonal variable spreading factor, the error is obtained by applying AWGN and $\mathrm{RN}$ were $25 \%$ and $18.75 \%$, respectively. This indicates that $\mathrm{RN}$ effect is less than the AWGN effect. However, in the PN, the error obtained by applying AWGN and RN are $37.5 \%$ and $31.25 \%$ respectively confirming that, $\mathrm{RN}$ effect is less than AWGN effect in the system. To conclude, the lowest effect of noise channel in the system will be achieved by applying the RN introduced by OVSF tree. The low cross-correlation standards between the codes is easy to the strain of a data message detection.

\section{REFERENCES}

[1] P. Singh, G. Soni, "Performance analysis of WCDMA link using QPSK \& QAM modulation schemes based on vector signal transceiver 5644R \& LabVIEW 2012", International Conference on Control, Instrumentation, Communication and Computational Technologies (ICCICCT), Kumaracoil, India, December 16-17, 2016

[2] M. Matthe, N. Michailow, I. Gaspar, G. Fettweis, "Influence of Pulse Shaping on Bit Error Rate Performance and Out of Band Radiation of Generalized Frequency Division Multiplexing", International Conference on Communications Workshops, Sydney, NSW, Australia, pp. 43-48, June 10-14, 2014

[3] N. Michailow, S.Krone, M. Lentmaier, G. Fettweis, "Bit Error Rate Performance of Generalized Frequency Division Multiplexing", $38^{\text {th }}$ IEEE - Vehicular Technology Conference (VTC Fall), Quebec, Canada, September 3-6, 2012

[4] P. Bah1, A. Farrago, I. Chlamtac, "Resource assignment for integrated service in wireless ATM networks", International Journal of Communication Systems, Vol. 11, No. 1, pp. 29-41, 1998

[5] M F. Alsharekh, M. Islam, A. H. Ibrahim, R. Khan, S. Habib, "Bit Error Rate Performance of RFID Signal in SDR Communication", Journal of Applied Sciences, Vol. 16, No. 4, pp. 161 -166, 2016

[6] T. Minn, K.-Y. Siu, "Dynamic assignment of orthogonal variable spreading factor codes in W-CDMA", IEEE. Journal on Selected Areas in Communications, Vol. 18, No. 8, pp. 1429-1440, 2000

[7] N. Cardona, A. Navarro, "W-CDMA capacity analysis using GIS based planning tools and MATLAB simulation", First International Conference on (Conf. Publ. No. 471) 3G Mobile Communication Technologies, London, UK, March 27-29, pp. 230-234, 2000

[8] C. W. WU, R. Q. Huang, "OVSF code management schemes on ad hoc networks", IEEE International Conference on Communications (IEEE Cat. No.04CH37577), Paris, France, Vol. 7, pp. 4152-4156, June20-24, 2004

[9] R. G. Winch, Telecommunication Transmission Systems: Microwave, Fiber Optic, Mobile Cellular Radio, Data, and Digital Multiplexing, McGraw-Hill, Inc., NY, USA, 1993 
[10] F. Liu, A.-J. Chen, C.-B. Xiang, H.-J. Song, "The intelligent monitoring method based on spectral correlation pseudo WCDMA", 4th International Conference on Computer Science and Network Technology, Harbin, China, pp. 1294-1298, December 19-20, 2015

[11] R. E. Ziemer, W. H. Tranter, Principles of Communications Systems Modulation and Noise, John Wiley \& Sons, 2014

[12] C. D'Amours, A. O. Dahmane, "Bit Error Rate Performance of a MIMOCDMA System Employing Parity-Bit-Selected Spreading in frequency Nonselective Rayleigh Fading”, International Journal of Antennas and Propagation, Vol. 2011, Article ID 516929, 2011

[13] M. Shen, G. Li, H. Liu, "Effect of traffic channel configuration on the orthogonal frequency division multiple access downlink performance", IEEE Transactions on Wireless Communications, Vol. 4, No. 4, pp. 1901-1913, 2005

[14] Z. Deng, Y. Liu, J. Liu, X. Chen, A. Argyriou, Z. Xu, S. Ci, "Crossnetwork and cross-layer optimized video streaming over LTE and WCDMA downlink", IEEE Symposium on Computers and Communication (ISCC), Messina, Italy, pp. 868-873, June 27-30, 2016

[15] Y.-C. Tseng, C.-M. Chao, "Code placement strategies for wideband CDMA OVSF code tree management", IEEE Transactions on Mobile Computing, Vol. 99, No. 4, pp. 293-302, 2002 